\title{
Overlapping Control Systems with Optimal Information Exchange
}

\author{
Somayeh Sojoudi ${ }^{\text {a }}$, Amir G. Aghdam ${ }^{\mathrm{b}}$ \\ ${ }^{a}$ Department of Control and Dynamical Systems, California Institute of Technology, Pasadena, CA 91125, USA \\ ${ }^{\mathrm{b}}$ Department of Electrical and Computer Engineering, Concordia University, Montréal, QC H3G 1M8, Canada
}

\begin{abstract}
This paper deals with the stabilizability of interconnected systems via linear time-invariant (LTI) decentralized controllers. Given a controllable and observable system with some distinct decentralized fixed modes (DFM), it is desired to find a suitable control structure (in terms of information flow) for it. Since a decentralized controller consists of a number of non-interacting local controllers, the objective here is to establish certain interactions between the local controllers in order to eliminate the undesirable DFMs. This objective is achieved by translating the knowledge of the system into some bipartite graphs. Then, the notions of minimal sets and maximal subgraphs are introduced, which lead to a simple combinatorial algorithm for solving the underlying problem. The efficacy of the results obtained is demonstrated by an illustrative example.
\end{abstract}

Key words: Decentralized control, Stabilization, Overlapping control

\section{Introduction}

Numerous real-world systems are composed of a number of interacting subsystems. The control of an interconnected system is often carried out by means of a set of local controllers, corresponding to the interacting subsystems (Davison et al., 1990; Siljak, 1991; Lavaei et al., 2006). It is sometimes assumed that the local controllers can fully communicate with each other in order to improve their effectiveness in a cooperative manner. However, this type of design technique is often problematic as the required data transmission between some of the local controllers can be very expensive, and sometimes infeasible. Consequently, it is normally desired that the local controllers either exchange partial information or act independently of each other. The latter case, where the overall controller consists of a set of isolated local controllers, is referred to as decentralized control in the literature (Ozguler, 2006; Davison et al., 1990; Siljak, 1991). The control structure in a decentralized control system is, in fact, block-diagonal.

\footnotetext{
^ This work has been supported by the Natural Sciences and Engineering Research Council of Canada under grant RGPIN-262127-07.

Email addresses: sojoudi@cds.caltech.edu (Somayeh Sojoudi), aghdam@ece.concordia.ca (Amir G. Aghdam).
}

The notion of a decentralized fixed mode (DFM) was introduced in Wang et al. (1973) to identify those modes of a system which are fixed with respect to any LTI decentralized controller. Various methods are introduced in the literature to characterize DFMs (Davison et al., 1990; Anderson et al., 1981; Anderson , 1982; Davison et al., 1985; Lavaei et al., 2007a). For instance, the method given in Davison et al. (1990) provides the existence conditions for DFMs in terms of the rank of a set of matrices. As a computationally more efficient technique, the papers Lavaei et al. (2007a,b) propose simple graphtheoretic approaches to verify whether an unrepeated mode of the system is a DFM.

Given an interconnected system with at least one unstable DFM, it is important to find out if a stabilizing LTI controller can be designed for the system by establishing new information flow channels in the control configuration. This question has been addressed in a number of papers to some extent by establishing communication links between certain local controllers. The work Armentano et al. (1982) uses this idea to tackle the underlying problem, but it fails to obtain the minimum number of required interactions to achieve stabilizability. This shortcoming limits the effectiveness of the method in practical applications considerably. The paper Unyelioglu et al. (1989) deals with the pole-assignability problem for the interconnected systems by means of partially inter- 
acting LTI local controllers. A cost is first attributed to the communication link between any pair of local controllers in order to formulate the implementation expenditure. However, the above-mentioned work considers only a particular class of modes, due to the complexity of the problem in the general case. This particular class includes only those fixed modes which result from the structure of the system, rather than an exact matching of the parameters of the system. Such fixed modes are referred to as structurally fixed modes or structurally decentralized fixed modes (SDFM) (Sezer et al., 1981). The method proposed in Unyelioglu et al. (1989) leads to a near-optimal solution by solving two separate optimization problems. A simpler method to handle the same problem (i.e., eliminating the SDFMs of a system) is more recently presented in Belmehdi et al. (2002). The work Zecevic et al. (2005) tackles the problem of eliminating the DFMs by introducing a low-rank centralized controller (i.e. interactions between all subsystems). The advantage of this method is that the controller obtained is robust. Although the above-mentioned work introduces several communication links, it attempts to justify the underlying idea by utilizing the notion of lowrank matrices.

This paper investigates the interconnected system with some distinct undesirable DFMs. A cost is assigned to each potential communication link between any pair of local controllers. This can, for instance, reflect the data transmission cost required for a communication link between the control stations. The ultimate goal can be described in two steps. The first step focuses on characterizing all the decentralized overlapping control structures with respect to which the system has no undesirable fixed modes. Then, in the second step, the optimal overlapping structure which minimizes the implementation cost (associated with establishing new links between local controllers) is determined. To this end, it is first shown that the unrepeated fixed modes of the system with respect to any overlapping control structure can be identified using a graph-theoretic approach. Then, the notions of minimal sets and maximal graphs are introduced to present a simple procedure for solving the problem under study. As a by-product of the proposed development, all the cost-effective decentralized overlapping control structures capable of eliminating the undesirable DFMs can also be characterized efficiently.

This paper is organized as follows. Some preliminary results are given in Section 2, which will later be used in the theoretical development. The main contributions of the paper are presented in Section 3. An illustrative example is given in Section 4 to demonstrate the significance and efficacy of this work. Finally, some concluding remarks are drawn in Section 5.

\section{Preliminaries}

Consider a LTI interconnected system $\mathcal{S}$ consisting of $\nu$ subsystems $S_{1}, S_{2}, \ldots, S_{\nu}$ with the following state-space representation:

$$
\begin{aligned}
\dot{x}(t) & =A x(t)+\sum_{j=1}^{\nu} B_{j} u_{j}(t) \\
y_{i}(t) & =C_{i} x(t)+\sum_{j=1}^{\nu} D_{i j} u_{j}(t), \quad i \in \bar{\nu}:=\{1, \cdots, \nu\}
\end{aligned}
$$

where $A$ is a matrix with $(i, j)$ block entry $A_{i j}$, for any $i, j \in \bar{\nu}$, and:

$$
\begin{aligned}
B_{j} & =\left[\begin{array}{llll}
B_{1 j}^{T} & B_{2 j}^{T} & \cdots & B_{\nu j}^{T}
\end{array}\right]^{T}, \\
C_{j} & =\left[\begin{array}{llll}
C_{j 1} & C_{j 2} & \cdots & C_{j \nu}
\end{array}\right], \quad j \in \bar{\nu}
\end{aligned}
$$

A structurally constrained controller for the system $\mathcal{S}$ consists of $\nu$ local controllers, partially interacting with each other. The following definition will prove convenient in formulating the interaction policy between local controllers.

Definition 1 Given a structurally constrained controller for the system $\mathcal{S}$, define the control interaction set $\mathbf{K}$ as a set which contains the entry $k_{i j}, i, j \in \bar{\nu}$, if and only if $y_{j}(t)$ can contribute to the construction of $u_{i}(t)$ in the controller.

By definition, the DFMs of $\mathcal{S}$ are those modes of the system which are fixed with respect to any LTI controller whose structure is specified by the control interaction set $\mathbf{K}_{d}:=\left\{k_{11}, k_{22}, \ldots, k_{\nu \nu}\right\}$ (Davison et al., 1990). Similarly, in the more general case of an overlapping controller with the interaction set $\mathbf{K}\left(\mathbf{K} \neq \mathbf{K}_{d}, \mathbf{K}_{d} \subset \mathbf{K}\right)$, the decentralized overlapping fixed modes (DOFM) of the system $\mathcal{S}$ w.r.t. $\mathbf{K}$ are those modes of the system which are fixed under any LTI controller whose structure complies with K (Lavaei et al., 2008).

\section{Main results}

Consider the system $\mathcal{S}$ given by (1), and assume that it has some distinct undesirable DFMs. It is desired to displace such fixed modes using a proper control structure. Let these undesirable modes be denoted by $\sigma_{1}, \sigma_{2}, \ldots, \sigma_{\mu}$. From the definition of DFMs, there is no LTI controller of a structure complying with $\mathbf{K}_{d}$ to displace any of these modes. Hence, it is desired to expand the control interaction set $\mathbf{K}_{d}$ by adding another set $\mathbf{K}_{e}$ to it such that none of these unwanted modes remains immovable with respect to the new control interaction set $\mathbf{K}_{d} \cup \mathbf{K}_{e}$. This problem is investigated in the sequel for a particular case first, and is extended to the general case, subsequently. 


\subsection{Displacing a single unrepeated DFM}

Assume that $\sigma$ is an arbitrary unrepeated mode of the system $\mathcal{S}$. One possible state-space realization for this system is given by:

$$
\begin{aligned}
\dot{\mathbf{x}}(t) & =\left[\begin{array}{ll}
\sigma & 0 \\
0 & \mathbf{A}
\end{array}\right] \mathbf{x}(t)+\sum_{j=1}^{\nu} \mathbf{B}_{j} u_{j}(t) \\
y_{i}(t) & =\mathbf{C}_{i} \mathbf{x}(t)+\sum_{j=1}^{\nu} \mathbf{D}_{i j} u_{j}(t), \quad i \in \bar{\nu}
\end{aligned}
$$

where the matrices $\mathbf{A}, \mathbf{B}_{j}, \mathbf{C}_{i}$ and $\mathbf{D}_{i j}, i, j \in \bar{\nu}$ can be obtained by using a proper similarity transformation, but their exact form is not important here (it is to be noted that DFMs are invariant under any similarity transformation). Define the matrix $M$ as follows:

$$
\begin{gathered}
M:=\left[\begin{array}{c}
\mathbf{C}_{1} \\
\vdots \\
\mathbf{C}_{\nu}
\end{array}\right]\left[\begin{array}{cc}
0 & 0 \\
0 & (\mathbf{A}-\sigma I)^{-1}
\end{array}\right]\left[\mathbf{B}_{1} \cdots \mathbf{B}_{\nu}\right]- \\
{\left[\begin{array}{ccc}
\mathbf{D}_{11} & \cdots & \mathbf{D}_{1 \nu} \\
\vdots & \ddots & \vdots \\
\mathbf{D}_{\nu 1} & \cdots & \mathbf{D}_{\nu \nu}
\end{array}\right]}
\end{gathered}
$$

Note that since the multiplicity of $\sigma$ is assumed to be 1 , it is not an eigenvalue of the matrix A. Denote the $(i, j)$ block entry of $M$ with $M_{i j} \in \Re^{r_{i} \times m_{j}}$, for any $i, j \in \bar{\nu}$.

A procedure is introduced now, to construct the graphs required to verify which modes of the system are DFMs.

Procedure 1 (Lavaei et al., 200\%a)

Construct a bipartite graph $\mathcal{G}$ with two sets of vertices $\mathcal{V}$ (set 1) and $\overline{\mathcal{V}}$ (set 2) and the tagged vertices $1,2, \ldots, \nu$ in each of the two sets. For any $i, j \in \bar{\nu}$, follow the steps below:

1) Connect vertex $j$ of the set $\mathcal{V}$ to vertex $i$ of the set $\overline{\mathcal{V}}$ if $M_{i j}=0$.

2) Mark vertex $i$ of the set $\mathcal{V}$ if the first column of the matrix $\mathbf{C}_{i}$ is a zero vector.

3) Mark vertex $j$ of the set $\overline{\mathcal{V}}$ if the first row of the matrix $\mathbf{B}_{j}$ is a zero vector.

Definition 2 Consider an arbitrary graph $\hat{\mathcal{G}}$ with $\zeta$ vertices labeled as vertex $1,2, \ldots, \zeta$ in any of its two sets. A subgraph of $\hat{\mathcal{G}}$ is said to span the vertices of $\hat{\mathcal{G}}$, if the labels of its vertices are distinct and form the set $\{1,2, \ldots, \zeta\}$.

Identify every subgraph of $\mathcal{G}$ which satisfies the following criteria: i) It is a complete bipartite subgraph.

ii) All of its vertices are marked.

iii) It spans the vertices of the graph $\mathcal{G}$.

Denote all such subgraphs with $\mathcal{G}_{1}, \mathcal{G}_{2}, \ldots, \mathcal{G}_{w}$. Moreover, denote set 1 and set 2 (see Procedure 1) of the graph $\mathcal{G}_{j}$ with $\mathcal{V}_{j}$ and $\overline{\mathcal{V}}_{j}$, respectively, for any $j \in\{1,2, \ldots, w\}$.

As an example, assume that the graph $\mathcal{G}$ for the mode $\sigma$ of a given system which is obtained from Procedure 1, is the one depicted in Figure 1. It can be easily observed from this graph that vertices 1 and 2 of the set $\mathcal{V}$ and vertices 3 and 4 of the set $\overline{\mathcal{V}}$ fulfill the three criteria pointed out above. Therefore, $\sigma$ is a DFM of the system (note that marked vertices are denoted by filled circles).

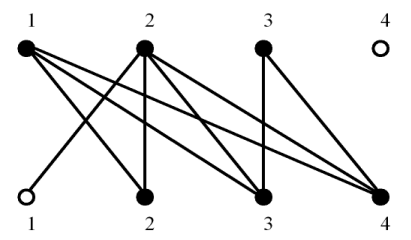

Fig. 1. The graph $\mathcal{G}$ of a given system.

The following lemma is borrowed from Lavaei et al. (2007a).

Lemma 1 The mode $\sigma$ is a DFM of the system $\mathcal{S}$ if and only if the nonnegative integer $w$ is strictly positive.

Assume for now that $w$ is strictly positive, and consequently the mode $\sigma$ is fixed with respect to any LTI controller complying with $\mathbf{K}_{d}$. It is desired to obtain all overlapping control structures capable of displacing this mode.

Procedure 2 For any given set $\left\{k_{i_{1} j_{1}}, k_{i_{2} j_{2}}, \ldots, k_{i_{z} j_{z}}\right\}$, form a bipartite graph $\mathcal{G}\left(\left\{k_{i_{1} j_{1}}, k_{i_{2} j_{2}}, \ldots, k_{i_{z} j_{z}}\right\}\right)$ as follows:

- Put $\nu+z$ vertices in set 1 and set 2 of the graph $\mathcal{G}\left(\left\{k_{i_{1} j_{1}}, \ldots, k_{i_{z} j_{z}}\right\}\right)$.

- Assign the labels $1,2, \ldots, \nu, j_{1}, j_{2}, \ldots, j_{z}$ to the vertices of set 1 .

- Assign the labels $1,2, \ldots, \nu, i_{1}, i_{2}, \ldots, i_{z}$ to the vertices of set 2.

- Consider any two arbitrary vertices of the graph $\mathcal{G}\left(\left\{k_{i_{1} j_{1}}, \ldots, k_{i_{z} j_{z}}\right\}\right)$ which do not pertain to the same set of vertices. Let the labels of these two vertices be $\lambda_{1}$ (in set 1) and $\lambda_{2}$ (in set 2). Connect these two vertices to each other in the graph $\mathcal{G}\left(\left\{k_{i_{1} j_{1}}, \ldots, k_{i_{z} j_{z}}\right\}\right)$ if and only if there is an edge between vertex $\lambda_{1}$ of $\mathcal{V}$ and vertex $\lambda_{2}$ of $\overline{\mathcal{V}}$ in the graph $\mathcal{G}$.

It is notable that some labels in the graph $\mathcal{G}\left(\left\{k_{i_{1} j_{1}}, k_{i_{2} j_{2}}\right.\right.$, $\left.\left.\ldots, k_{i_{z} j_{z}}\right\}\right)$ are recurrent. The next theorem proposes a simple method to verify whether or not the mode $\sigma$ is a 
DOFM of the system $\mathcal{S}$ with respect to a given control interaction set.

Theorem 1 Given the set $\left\{k_{p_{1} q_{1}}, k_{p_{2} q_{2}}, \ldots, k_{p_{\alpha} q_{\alpha}}\right\}$, the mode $\sigma$ is not a DOFM of the system w.r.t. $\mathbf{K}_{d} \cup$ $\left\{k_{p_{1} q_{1}}, \ldots, k_{p_{\alpha} q_{\alpha}}\right\}$ if and only if the graph $\mathcal{G}\left(\left\{k_{p_{1} q_{1}}, \ldots\right.\right.$, $\left.\left.k_{p_{\alpha}} q_{\alpha}\right\}\right)$ does not contain a complete bipartite subgraph with all vertices marked, which spans its vertices (see Definition 2).

Sketch of the proof: The proof will be given here for $\alpha=$ 1 , as its generalization is straightforward. By obtaining two transformation matrices discussed in Lavaei et al. (2008) and pursuing the approach given therein, it can be easily verified that $\sigma$ is a DOFM of the system $\mathcal{S}$ w.r.t. the control interaction set $\mathbf{K}_{d} \cup\left\{k_{p_{1} q_{1}}\right\}$ if and only if it is a DFM of the following system:

$$
\begin{aligned}
& \dot{\tilde{x}}(t)=A \tilde{x}(t)+\sum_{j=1}^{\nu} B_{j} \tilde{u}_{j}(t)+B_{p_{1}} \tilde{u}_{\nu+1}(t) \\
& \tilde{y}_{i}(t)=C_{i} \tilde{x}(t)+\sum_{j=1}^{\nu} D_{i j} \tilde{u}_{j}(t)+D_{i p_{1}} \tilde{u}_{\nu+1}(t), \quad i \in \bar{\nu} \\
& \tilde{y}_{\nu+1}(t)=\tilde{C}_{q_{1}} \tilde{x}(t)+\sum_{j=1}^{\nu} D_{q_{1} j} \tilde{u}_{j}(t)+D_{q_{1} p_{1}} \tilde{u}_{\nu+1}(t)
\end{aligned}
$$

(note that the above system has one input and one output more than the system $\mathcal{S}$ ). The proof follows by applying the graph-theoretic approach provided in Lavaei et al. (2007a) (which was explained in Lemma 1 for the system $\mathcal{S}$ ) to the system given in $(5)$.

So far, it is shown how the existence of a DOFM can be concluded from a bipartite graph associated with the system. This result will be used next to characterize all desirable overlapping control structures.

Definition 3 The set $\left\{k_{p_{1} q_{1}}, k_{p_{2} q_{2}}, \ldots, k_{p_{\alpha} q_{\alpha}}\right\}$ corresponding to the system $\mathcal{S}$ is said to be minimal w.r.t. $\sigma$ if and only if the mode $\sigma$ is not a DOFM of $\mathcal{S}$ w.r.t. $\mathbf{K}_{d} \cup\left\{k_{p_{1} q_{1}}, k_{p_{2} q_{2}}, \ldots, k_{p_{\alpha} q_{\alpha}}\right\}$, while it is a DOFM of $\mathcal{S}$ w.r.t. $\mathbf{K}_{d} \cup\left\{k_{p_{1} q_{1}}, \ldots, k_{p_{j-1} q_{j-1}}, k_{p_{j+1} q_{j+1}}, \ldots, k_{p_{\alpha} q_{\alpha}}\right\}$ for any $j \in\{1,2, \ldots, \alpha\}$.

Definition 4 A subgraph of the graph $\mathcal{G}$ corresponding to the system $\mathcal{S}$ is said to be maximal if the following four conditions hold:

i) It is a complete bipartite subgraph.

ii) All of its vertices are marked.

iii) The set of the labels of its vertices is equal to the set $\bar{\nu}$.

iv) The graph $\mathcal{G}$ has no other subgraph satisfying criteria (i), (ii) and (iii) given above such that it includes this subgraph.

Using proper combinatorial algorithms, the maximal subgraphs of $\mathcal{G}$ can be easily identified (analogously to the algorithms for finding the complete bipartite graphs with maximum number of edges). Denote such subgraphs with $\tilde{\mathcal{G}}_{1}, \tilde{\mathcal{G}}_{2}, \ldots, \tilde{\mathcal{G}}_{\tilde{w}}$. Moreover, denote set 1 and set 2 of vertices of the graph $\tilde{\mathcal{G}}_{j}$ with $\tilde{\mathcal{V}}_{j}$ and $\overline{\tilde{V}}_{j}$, respectively, for any $j \in\{1,2, \ldots, \tilde{w}\}$. It is to be noted that the number $\tilde{w}$ is typically small, due to the generic property of the fixed modes. The following remark aims to present a bound on the number $\tilde{w}$.

Remark 1 From the definition of a maximal graph, the sets of vertices $\tilde{\mathcal{V}}_{1}, \ldots, \tilde{\mathcal{V}}_{\tilde{w}}$ are all distinct. Moreover, it is straightforward to show that one of the sets $\mathcal{V}_{1}, \mathcal{V}_{2}, \ldots, \mathcal{V}_{w}$ is exactly the same as $\tilde{\mathcal{V}}_{j}$, for any $j \in\{1,2, \ldots, \tilde{w}\}$. These two facts point to the inequality $\tilde{w} \leq w$.

Theorem 2 Assume that the set $\left\{k_{p_{1} q_{1}}, k_{p_{2} q_{2}}, \ldots, k_{p_{\alpha} q_{\alpha}}\right\}$ is minimal w.r.t. the mode $\sigma$. Then, the number $\alpha$ is less than or equal to $\tilde{w}$.

Proof: From the definition of a minimal set, the mode $\sigma$ is a DOFM of the system $\mathcal{S}$ w.r.t. the control interaction set $\mathbf{K}_{d} \cup\left\{k_{p_{1} q_{1}}, \ldots, k_{p_{j-1} q_{j-1}}, k_{p_{j+1} q_{j+1}}, \ldots, k_{p_{\alpha} q_{\alpha}}\right\}$, for any $j \in\{1,2, \ldots, \alpha\}$. Hence, it can be concluded from Theorem 1 that the graph $\mathcal{G}\left(\left\{k_{p_{1} q_{1}}, \ldots, k_{p_{j-1} q_{j-1}}, k_{p_{j+1} q_{j+1}}\right.\right.$, $\left.\ldots, k_{p_{\alpha} q_{\alpha}}\right\}$ ) has a complete bipartite subgraph with marked vertices, which span the vertices of said graph. This subgraph should include either the duplicated vertex $q_{i}$ in its set 1 or the duplicated vertex $p_{i}$ in its set 2 , for all $i \in\{1,2, \ldots, j-1, j+1, \ldots, \alpha\}$. On the other hand, it is straightforward to show that there exists an integer $f_{j} \in\{1, \ldots, \tilde{w}\}$ such that this subgraph is included in $\tilde{\mathcal{G}}_{f_{j}}$ (in light of the definition of a maximal graph). Thus, one comes to the conclusion immediately that the following logic statement is true:

$\left(q_{i} \in \tilde{\mathcal{V}}_{\left(f_{j}\right)}\right) \vee\left(p_{i} \in \overline{\tilde{\mathcal{V}}}_{\left(f_{j}\right)}\right), \forall i \in\{1,2, \ldots, j-1, j+1, \ldots, \alpha\}$

where $\vee$ is the logic $O R$ operation. Now, to prove Theorem 2 by contradiction, assume that $\tilde{w}<\alpha$. Since all the natural numbers $f_{1}, f_{2}, \ldots, f_{\alpha}$ belong to the set $\{1,2, \ldots, \tilde{w}\}$ and also the inequality $\tilde{w}<\alpha$ holds, it can be concluded from Dirichlet's principle that at least two of the values $f_{1}, f_{2}, \ldots, f_{\alpha}$ are identical. Without any loss of generality, assume that $f_{1}=f_{2}=f$ for some positive number $f$. Consider the statement (6) for the values $j=1$ and $j=2$. The amalgamation of these two sets of relations will arrive at the following true statement:

$$
\left(q_{i} \in \tilde{\mathcal{V}}_{f}\right) \vee\left(p_{i} \in \overline{\tilde{\mathcal{V}}}_{f}\right), \forall i \in\{1,2, \ldots, \alpha\}
$$

The above logic statement yields that the graph $\mathcal{G}\left(\left\{k_{p_{1} q_{1}}, k_{p_{2} q_{2}}, \ldots, k_{p_{\alpha} q_{\alpha}}\right\}\right)$ includes a complete bipartite subgraph with the properties pointed out in Theorem 1. This implies that the mode $\sigma$ is a DOFM w.r.t. $\mathbf{K}_{d} \cup\left\{k_{p_{1} q_{1}}, k_{p_{2} q_{2}}, \ldots, k_{p_{\alpha} q_{\alpha}}\right\}$, which contradicts the original assumption of minimality. 
Theorem 2 states that if by adding more than $\tilde{w}$ communication links to the decentralized control structure the mode $\sigma$ is no longer fixed, then some of the links are redundant and have no essential contribution in displacing the mode. One can use the following theorem to develop an algorithm for finding the minimal sets systematically.

Theorem 3 The set $\left\{k_{p_{1} q_{1}}, k_{p_{2} q_{2}}, \ldots, k_{p_{\alpha} q_{\alpha}}\right\}$ is minimal w.r.t. $\sigma$ if and only if the criteria given below both hold:

i) For any $j \in\{1,2, \ldots, \alpha\}$, there exists an integer $f_{j} \in$ $\{1, \ldots, \tilde{w}\}$ such that the statements:

$$
\begin{aligned}
& \left(q_{i} \in \tilde{\mathcal{V}}_{\left(f_{j}\right)}\right) \vee\left(p_{i} \in \overline{\tilde{\mathcal{V}}}_{\left(f_{j}\right)}\right), \forall i \in\{1, \ldots, \alpha\} \backslash\{j\} \\
& \left(q_{j} \notin \tilde{\mathcal{V}}_{\left(f_{j}\right)}\right) \wedge\left(p_{j} \notin \overline{\tilde{\mathcal{V}}}_{\left(f_{j}\right)}\right)
\end{aligned}
$$

are true, where $f_{1}, f_{2}, \ldots, f_{\tilde{w}}$ are all distinct (note that $\wedge$ is the logic $A N D$ operation).

ii) There exists no integer $f \in\{1, \ldots, \tilde{w}\}$ such that the following logic statement is true:

$$
\left(q_{i} \in \tilde{\mathcal{V}}_{f}\right) \vee\left(p_{i} \in \overline{\tilde{\mathcal{V}}}_{f}\right), \forall i \in\{1,2, \ldots, \alpha\}
$$

Proof: The proof of this theorem follows directly from the discussion given in the proof of Theorem 2 .

Theorem 3 implicitly proposes a simple method to compute all the minimal sets w.r.t. the fixed mode $\sigma$.

Remark 2 Although graph-based problems are computationally intractable in general and hence very difficult to solve, the graph-theoretic part of the technique proposed here has a very particular form and the existing sum-of-square (SOS) methods can be employed to efficiently handle it. This can be carried out, for instance, in line with the ideas used in Parrilo (2000) for solving the $M A X-C U T$ problem in graph theory.

\subsection{Displacing multiple unrepeated DFMs}

The methodology presented in the preceding subsection will now be deployed to characterize all the control interaction sets $\mathbf{K}_{e}$ such that the DFMs $\sigma_{1}, \sigma_{2}, \ldots, \sigma_{\mu}$ are all movable w.r.t to $\mathbf{K}_{d} \cup \mathbf{K}_{e}$. Note that although by assumption the mode $\sigma_{i}, i \in\{1,2, \ldots, \mu\}$, is an unrepeated DFM of the system $\mathcal{S}$, its multiplicity as a regular mode of the system can be greater than 1 . In such cases, the aforementioned method cannot be applied to the system directly. As a remedy for this problem, one can consider a generic static decentralized controller and apply it to the system $\mathcal{S}$ so that the multiplicity of the mode $\sigma_{i}$, $i \in\{1,2, \ldots, \mu\}$, becomes exactly equal to 1 in the resultant system (Davison et al., 1990; Aghdam et al., 2008). Therefore, with no loss of generality, assume henceforth that the mode $\sigma_{i}, i \in\{1,2, \ldots, \mu\}$, is not only an unrepeated DFM of the system $\mathcal{S}$, but also an unrepeated mode of it.
For any $i \in\{1,2, \ldots, \mu\}$, obtain all minimal sets associated with the mode $\sigma_{i}$ using the approach given in the previous subsection, and denote them with $\mathbf{K}_{e}^{i, 1}, \mathbf{K}_{e}^{i, 2}, \ldots, \mathbf{K}_{e}^{i, z_{i}}$. The following corollary states how the underlying problem can be treated.

Corollary 1 Given the control interaction set $\mathbf{K}_{e}$, none of the modes $\sigma_{1}, \sigma_{2}, \ldots, \sigma_{\mu}$ are DOFMs of the system $\mathcal{S}$ w.r.t. $\mathbf{K}_{d} \cup \mathbf{K}_{e}$ if and only if there exist integers $g_{1}, g_{2}, \ldots, g_{\mu}$ with the following property:

$$
\left\{\mathbf{K}_{e}^{1, g_{1}} \cup \mathbf{K}_{e}^{2, g_{2}} \cup \cdots \cup \mathbf{K}_{e}^{\mu, g_{\mu}}\right\} \subseteq \mathbf{K}_{e}
$$

Proof: The proof follows immediately from the definition of a minimal set.

In practice, it is desired that the addition of the set of communication links $\mathbf{K}_{e}$ to the control structure be as inexpensive as possible. In order to take the cost of the added links into account, it is assumed that the cost of implementing the communication link $k_{i j}$ is denoted by $\mathcal{C}_{i j}$, for any $i, j \in \bar{\nu}$. Note that this cost is normally related to the factors such as the distance between the subsystems or the nature of the outputs to be transmitted to the other subsystems. This restriction results from the fact that different state-space representations are used throughout the paper for different modes, which may lead to the inconsistency in the cost evaluation. By virtue of Corollary 1, the problem of finding the least costly $\mathbf{K}_{e}$ with the aforementioned property (i.e., displacing certain fixed modes) can be reformulated as the problem of obtaining all the sets $\mathbf{K}_{e}$ representable as $\mathbf{K}_{e}^{1, g_{1}} \cup \mathbf{K}_{e}^{2, g_{2}} \cup \cdots \cup \mathbf{K}_{e}^{\mu, g_{\mu}}$ for some integers $g_{1}, g_{2}, \ldots, g_{\mu}$, and computing their associated costs accordingly to determine which one is the least expensive.

Remark 3 It may turn out that communication channels between some of the subsystems cannot be established by any means. In order to take this constraint into account, two strategies can be pursued. First, one can assign sufficiently large cost values to impermissible links so that they do not appear in the optimal configuration. Alternatively, one can first obtain all the minimal sets, and then exclude the ones containing inadmissible channels. In other words, the optimization must be carried out over the minimal sets with permissible elements.

\section{Numerical example}

Let $\mathcal{S}$ be a system consisting of four single-input singleoutput (SISO) subsystems with the following decoupled 
state-space matrices:

$$
\begin{aligned}
& A=\left[\begin{array}{llll}
1 & 0 & 0 & 0 \\
0 & 2 & 0 & 0 \\
0 & 0 & 3 & 0 \\
0 & 0 & 0 & 4
\end{array}\right], B=\left[\begin{array}{llll}
3 & 0 & 0 & 0 \\
4 & 2 & 7 & 0 \\
0 & 0 & 9 & 8 \\
1 & 6 & -5 & 7
\end{array}\right] \\
& C=\left[\begin{array}{llll}
0 & 2 & 4 & 3 \\
0 & -6 & 0 & 8 \\
0 & 4 & 0 & -9 \\
5 & 1 & 0 & 7
\end{array}\right], D=\left[\begin{array}{cccc}
-5 & 10 & 27 & 23 \\
32 & 60 & -3 & 56 / 3 \\
-25 & -62 & 43 & -21 \\
-4.5 & 40 & 16 & 7
\end{array}\right]
\end{aligned}
$$

Consider the mode $\sigma=1$, whose corresponding graph $\mathcal{G}$ is depicted in Figure 2(a). This graph contains a complete bipartite subgraph consisting of vertex 1 from set 1 of $\mathcal{G}$ and vertices 2,3 and 4 from set 2 of $\mathcal{G}$ which are all marked. This subgraph spans the vertices of $\mathcal{G}$, and hence it results from Lemma 1 that the mode $\sigma=1$ is a DFM of the system. Likewise, it can be shown that the mode $\sigma=3$ is also a DFM of the system, while the modes 2 and 4 are not (note that the control interaction set corresponding to a decentralized controller in this example is equal to $\left.\mathbf{K}_{d}=\left\{k_{11}, k_{22}, k_{33}, k_{44}\right\}\right)$.

It is desired now to expand the structure of the controller from decentralized to overlapping so that both of the unstable DFMs can be displaced. Let the mode $\sigma=1$ be treated first. It is straightforward to observe from the graph $\mathcal{G}$ in Figure $2(\mathrm{a})$ that $\tilde{w}=2$. Therefore, the subgraphs $\mathcal{G}_{1}$ and $\mathcal{G}_{2}$ will have the following sets of vertices:

$$
\mathcal{V}_{1}=\{1\}, \overline{\mathcal{V}}_{1}=\{2,3,4\}, \mathcal{V}_{2}=\{1,2,3\}, \overline{\mathcal{V}}_{2}=\{4\}
$$

On the other hand, the graph $\mathcal{G}$ has two maximal subgraphs which are the same as $\mathcal{G}_{1}$ and $\mathcal{G}_{2}$. It can now be concluded from Theorem 3 that the minimal sets w.r.t. $\sigma=1$ are:

$$
\mathbf{K}_{e}^{1,1}=\left\{k_{14}\right\}, \mathbf{K}_{e}^{1,2}=\left\{k_{12}, k_{34}\right\}, \mathbf{K}_{e}^{1,3}=\left\{k_{13}, k_{24}\right\}
$$

Note that as expected from Theorem 2, these sets have at most 2 elements, due to the relation $\tilde{w}=2$. Analogously, the minimal sets w.r.t. $\sigma=3$ can be obtained as:

$$
\mathbf{K}_{e}^{3,1}=\left\{k_{31}\right\}, \quad \mathbf{K}_{e}^{3,2}=\left\{k_{41}\right\}
$$

(note that for this mode, $\tilde{w}$ is equal to 1 ). It results from Corollary 1 that the modes 1 and 3 are not DOFMs of the system w.r.t. the control interaction $\mathbf{K}_{d} \cup \mathbf{K}_{e}$ if and only if the following condition is satisfied for the set $\mathbf{K}_{e}$ :

$$
\exists \zeta_{1} \in\{1,2,3\}, \exists \zeta_{2} \in\{1,2\}: \quad\left\{\mathbf{K}_{e}^{1, \zeta_{1}} \cup \mathbf{K}_{e}^{3, \zeta_{2}}\right\} \subseteq \mathbf{K}_{e}
$$

Assume first that all of the communication links have the same cost, i.e., $\mathcal{C}_{i j}=1, i, j \in\{1,2,3,4\}$. In this case, the least costly interaction sets would be $\left\{k_{14}, k_{31}\right\}$ and $\left\{k_{14}, k_{41}\right\}$ with the implementation cost of 2 . The graph $\mathcal{G}\left(\left\{k_{14}, k_{31}\right\}\right)$ is depicted in Figures $2(\mathrm{~b})$ for $\sigma=1$, and in Figure 2(c) for $\sigma=3$. It can be easily verified that none of these graphs has a subgraph with the properties mentioned in Theorem 1. This confirms the result that the modes 1 and 3 are not the DOFMs of the system $\mathcal{S}$ w.r.t. $\mathbf{K}_{d} \cup\left\{k_{14}, k_{31}\right\}$.
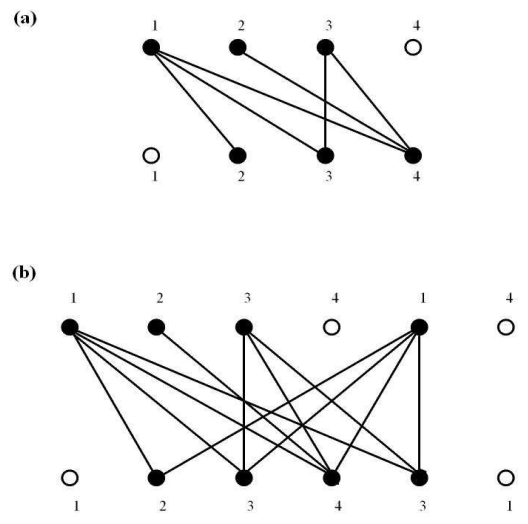

(c)

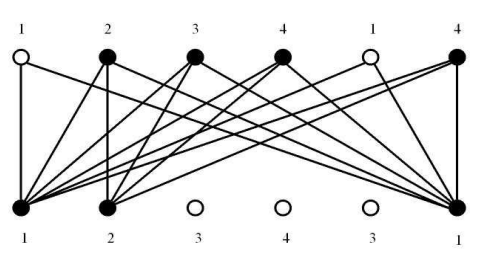

Fig. 2. a) The graph $\mathcal{G}$ associated with the mode $\sigma=1$; b) the graph $\mathcal{G}\left(\left\{k_{14}, k_{31}\right\}\right)$ corresponding to the mode $\sigma=1$; c) the graph $\mathcal{G}\left(\left\{k_{14}, k_{31}\right\}\right)$ corresponding to the mode $\sigma=3$.

Now, consider a different implementation cost as follows:

$$
\begin{aligned}
& \mathcal{C}_{14}=5, \quad \mathcal{C}_{12}=2, \quad \mathcal{C}_{34}=2, \quad \mathcal{C}_{13}=1, \\
& \mathcal{C}_{24}=1, \quad \mathcal{C}_{31}=5, \quad \mathcal{C}_{41}=4
\end{aligned}
$$

In this case, the optimal set $\mathbf{K}_{e}$ would be $\left\{k_{13}, k_{24}, k_{41}\right\}$ with the implementation cost of 6 .

\section{Conclusions}

This paper tackles the stabilizability problem for an interconnected system with a number of distinct undesirable decentralized fixed modes (DFM), by means of the structurally constrained controllers. It is well-known that a linear time-invariable (LTI) decentralized controller comprising a set of isolated local controllers cannot displace any DFMs. Thus, the objective of this paper is to establish some interactions between the local controllers in order to displace the undesirable DFMs. To this end, the knowledge of the system is transformed into a number of bipartite graphs (corresponding to the 
unwanted DFMs). Subsequently, the notions of minimal sets of interactions and maximal subgraphs are introduced. A simple procedure is then proposed to characterize all the possible sets of interactions which can displace the undesirable DFMs. The numerical example provided elucidates the efficacy of the results.

\section{Acknowledgements}

The authors would like to gratefully acknowledge the insightful comments of Mr. Javad Lavaei from the California Institute of Technology, which were essential in the development of the main results of the paper.

\section{References}

Aghdam, A. G., Davison, E. J. (2008). Discrete-Time Control of Continuous Systems with Approximate Decentralized Fixed Modes. Automatica, 1, pp. 7587.

Anderson, B. D. O., Clements, D. J. (1981). Algebraic characterizations of fixed modes in decentralized systems. Automatica 17(5), pp. 703-712.

Anderson, B. D. O. (1982). Transfer function matrix description of decentralized fixed modes. IEEE Transactions on Automatic Control, 27(6), pp. 11761182.

Armentano, V., Singh, M. (1982). A procedure to eliminate decentralized fixed modes with reduced information exchange. IEEE Transactions on Automatic Control, 27(1), pp. 258-260.

Belmehdi, A., Boukhetala, D. (2002). Method to eliminate structurally fixed modes in decentralized control systems. International Journal of Systems Science, 33(15), pp. 1249-1256.

Davison, E. J., Wang, S. H. (1985). A characterization of decentralized fixed modes in terms of transmission zeros. IEEE Transactions on Automatic Control, 30(1), pp. 81-82.

Davison, E. J., Chang, T. N. (1990). Decentralized stabilization and pole assignment for general proper systems. IEEE Transactions on Automatic Control, $35(6)$, pp. $652-664$.

Gong, Z., Aldeen, M. (1997). Stabilization of decentralized control systems. Journal of Mathematical Systems, Estimation, and Control, 7(1), pp. 1-16.

Lavaei, J., Aghdam, A. G. (2008). Control of continuoustime LTI systems by means of structurally constrained controllers. Automatica, 1, pp. 141-148.

Lavaei, J., Aghdam, A. G. (2006). Decentralized control design for interconnected systems based on a centralized reference controller. Proceedings of the 45th IEEE Conference on Decision and Control, San Diego, CA, pp. 1189-1195.

Lavaei, J., Aghdam, A. G. (2007a). Characterization of decentralized and quotient fixed modes via graph theory. Proceedings of the 2007 American Control Conference, New York, NY, pp. 790-795.
Lavaei, J., Aghdam, A. G. (2007b). A Graph Theoretic Method to Find Decentralized Fixed Modes of LTI Systems. Automatica, 43(2), pp. 2129-2133.

Özgüler, A. B. (2006). Global stabilization via local stabilizing actions. IEEE Transactions on Automatic Control, 51(3), pp. 530-533.

Parrilo, P. A. (2000). Structured semidefinite programs and semialgebraic geometry methods in robustness and optimization. Ph.D. thesis, California Institute of Technology, (available at http://www.mit.edu/ parrilo/).

Sezer, M. E., Šiljak, D. D. (1981). Structurally fixed modes. Systems \& Control Letters, 1(1), pp. 60-64.

Šiljak, D. D. (1991). Decentralized control of complex system. Cambridge: Academic Press.

Unyelioglu, K., Sezer, E. (1989). Optimum feedback patterns in multivariable control systems. International Journal of Control, 49(3), pp. 791-808.

Wang, S. H., Davison, E. J. (1973). On the stabilization of decentralized control systems. IEEE Transactions on Automatic Control, 18(5), pp. 473-478.

Zecevic, A. I., Šiljak, D. D. (2005). Global low-rank enhancement of decentralized control for large-scale systems. IEEE Transactions on Automatic Control, 50(5), pp. 740-744. 